\title{
ROBUST MOSAICKING OF UAV IMAGES WITH NARROW OVERLAPS
}

\author{
J. Kim ${ }^{\text {a }}$, T. Kim ${ }^{\text {a, } * \text {, D. Shin }}$, S.H. Kim ${ }^{\text {b }}$ \\ ${ }^{a}$ Dept. of Geoinformatic Engineering, Inha University, 100 Inharo, Namgu, Incheon, Korea - \\ jikim3124@inha.edu, tezid@inha.ac.kr \\ bAgency for Defense Development, Yuseong, Daejeon, Korea - (dsshin, falcon)@add.re.kr
}

Commission I, ICWG I/Vb

KEY WORDS: Image Mosaicking, Geometric correction, UAV Images, Narrow overlaps

\begin{abstract}
:
This paper considers fast and robust mosaicking of UAV images under a circumstance that each UAV images have very narrow overlaps in-between. Image transformation for image mosaicking consists of two estimations: relative transformations and global transformations. For estimating relative transformations between adjacent images, projective transformation is widely considered. For estimating global transformations, panoramic constraint is widely used. While perspective transformation is a general transformation model in 2D-2D transformation, this may not be optimal with weak stereo geometry such as images with narrow overlaps. While panoramic constraint works for reliable conversion of global transformation for panoramic image generation, this constraint is not applicable to UAV images in linear motions. For these reasons, a robust approach is investigated to generate a high quality mosaicked image from narrowly overlapped UAV images. For relative transformations, several transformation models were considered to ensure robust estimation of relative transformation relationship. Among them were perspective transformation, affine transformation, coplanar relative orientation, and relative orientation with reduced adjustment parameters. Performance evaluation for each transformation model was carried out. The experiment results showed that affine transformation and adjusted coplanar relative orientation were superior to others in terms of stability and accuracy. For global transformation, we set initial approximation by converting each relative transformation to a common transformation with respect to a reference image. In future work, we will investigate constrained relative orientation for enhancing geometric accuracy of image mosaicking and bundle adjustments of each relative transformation model for optimal global transformation.
\end{abstract}

\section{INTRODUCTION}

Image mosaicking aims to generate a seamless composite image, which is geometrically and radiometrically consistent, from a set of overlapping images. For this, geometric relationships among images need to be determined. After geometric correction from the relationships is performed, radiometric and geometric inconsistency occurred between adjacent images is then minimized by radiometric correction, seamline extraction and image blending.

Among geometric correction schemes, the most accurate result can be achieved by ortho-rectification scheme using 3D terrain model of a target area. This scheme generally needs image matching process or precise 3D terrain dataset. However, this requirement may be limited in certain applications. In this cases, image-to-image based geometric correction that additional information for the target area is not required can be preferred for image mosaicking.

In this approach, quality of a mosaicked image widely depends on accuracy of the geometric relationships and transformations among overlapping images. Image transformation consists of two estimations: relative transformations and global transformations. For estimating relative transformations between adjacent images, projective transformation is widely considered. For estimating global transformations, panoramic constraint is widely used. However, these cannot be applied to our situation. While the perspective transformation is a general transformation model in 2D-2D plane transformation, this may not be optimal with weak stereo geometry such as images with narrow overlaps. While the panoramic constraint works for reliable conversion of global transformation for panoramic image generation (Brown and Lowe, 2007), this constraint is not applicable to UAV (unmanned aircraft vehicle) images in linear motions. For these reasons, we investigate a robust approach to generate a high quality mosaicked image from narrowly overlapped UAV images. In this paper, we particularly focus on the geometric correction part for the whole image mosaicking process. In order to identify optimal geometric correction method in the case of narrowly overlapping images, several transformation models are discussed and evaluated.

\section{GEOMETRIC CORRECTION FOR NARROWLY OVERLAPPING IMAGES}

Image-to-image based geometric correction consists of relative transformation and global transformation. Each corrected image constructing mosaicked image is generated by global transformation derived from relative transformation relationships between adjacent images. In the following subsections, relative and global transformations proposed for robust image mosaicking are described.

\subsection{Relative transformation}

Global transformations of each image for image mosaicking are derived from relative transformations among adjacent images. Therefore, in order to generate high quality mosaicked image, high geometric accuracy of relative transformations is needed to

\footnotetext{
* Corresponding author
} 
be ensured. In case of weak geometry with narrow overlaps, stability or reliability of geometry estimation also has to be addressed. For this reason, we consider several transformation models as relative transformation. Among them were perspective transformation, affine transformation, coplanar relative orientation, and relative orientation with reduced adjustment parameters.

First one is affine transformation. This model describes scale, rotation, translation, and skew between two 2D planes in 2D space. Although this model is not enough to explain 3D motions between two image planes, there is a merit in terms of stability of geometry estimation.

$$
\left[\begin{array}{c}
x_{i}^{\prime} \\
y_{i}^{\prime} \\
1
\end{array}\right]=\left[\begin{array}{lll}
a & b & c \\
d & e & f \\
0 & 0 & 1
\end{array}\right]\left[\begin{array}{c}
x_{i} \\
y_{i} \\
1
\end{array}\right]
$$

Second one is perspective (or projective) transformation. Since this transformation model can explain general motions between two image planes in 3D space, this has been generally used in many applications.

$$
s_{i}\left[\begin{array}{c}
x_{i}^{\prime} \\
y_{i}^{\prime} \\
1
\end{array}\right] \sim\left[\begin{array}{lll}
h_{11} & h_{12} & h_{13} \\
h_{21} & h_{22} & h_{23} \\
h_{31} & h_{32} & h_{33}
\end{array}\right]\left[\begin{array}{c}
x_{i} \\
y_{i} \\
1
\end{array}\right]
$$

In computer vision field, this transformation model called homography is estimated by Levenberg-Marquardt (LM) method minimizing reprojection errors as

$$
\begin{aligned}
& e=\sum_{i}\left\{\left(x_{i}^{\prime}-\frac{h_{11} x_{i}+h_{12} y_{i}+h_{13}}{h_{31} x_{i}+h_{32} y_{i}+h_{33}}\right)^{2}\right. \\
&\left.+\left(y_{i}^{\prime}-\frac{h_{21} x_{i}+h_{22} y_{i}+h_{23}}{h_{31} x_{i}+h_{32} y_{i}+h_{33}}\right)^{2}\right\}
\end{aligned}
$$

On the other hand, in photogrammetry field, this perspective transformation is estimated by relative orientation method based on coplanarity (or collinearity) condition. In this paper, this approach is named as standard coplanar relative orientation to differentiate from the computer vision approach.

$$
\mathbf{H}=\mathbf{K}\left(\mathbf{R}+\mathbf{T n}^{\mathrm{t}} / d\right) \mathbf{K}^{-1}
$$

where $\mathbf{R}, \mathbf{T}, \mathbf{K}$ are rotation, translation and camera matrix respectively. $\mathbf{n}$ a normal vector, and $d$ the distance from the perspective center to a reference plane, and $\mathbf{H}$ homography for the perspective transformation. This approach assumes that intrinsic parameters of camera such as focal length, principle point, lens distortion coefficients, etc., were known. Extrinsic parameters of each camera are estimated as unknowns. Transformation is then calculated from the camera parameters.

In the standard coplanar relative orientation approach, characteristics of flight geometry are easily reflected by adjusting orientation parameters. In general, UAV linearly moves along with flight direction. This indicates that flight motion of UAV can be more simply modelled than general configuration as Figure 1(a). Therefore, we can reduce unknown parameters for coplanar relative orientation as Figure 1(b). This parameter adjustment can contribute to increasing stability of geometry estimation.

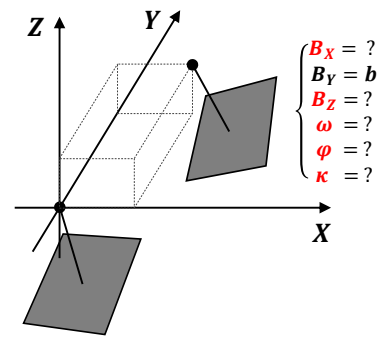

(a) General configuration

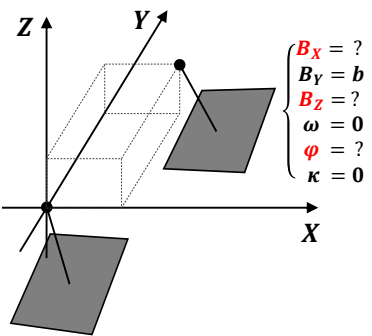

(b) Adjusted configuration
Figure 1. Perspective transformation estimation considering characteristics of flight geometry.

\subsection{Global transformation}

Global transformations define mapping each image plane into mosaicked image plane. These are derived from relative transformations among adjacent images. For determining the global transformations of each image, maximum spanning tree and breadth-first search algorithms are applied as explained in Figure 2 (Brown and Lowe, 2007). By the maximum spanning tree generated from number of tiepoints between adjacent images, best image pairs with strong stereo geometry are determined. By breadth-first search, global transformations of each image are determined. This breadth-first searching began from finding base image to make the lowest depth of image tree and the highest connectivity with adjacent images. These contribute to reducing accumulated projection errors occurred by calculating global transformations of each image from relative transformations.

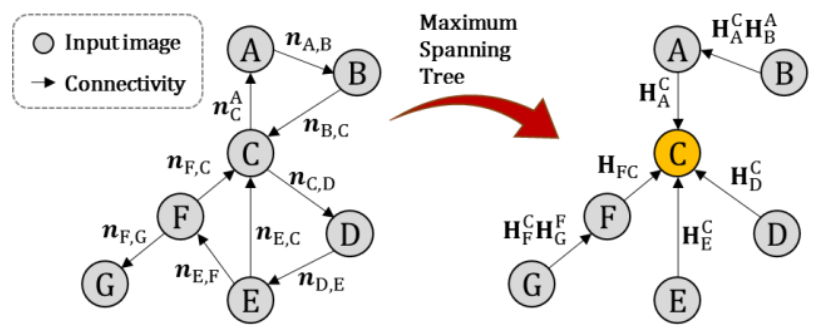

Figure 2. Determination of global transformation from relative transformation.

\section{PERFORMANCE EVALUATION}

Performance evaluation for each transformation model was carried out using two strips of UAV images (image size: $4272 \times 2848$ pixels, focal length: $40 \mathrm{~mm}$, pixel size: $4.3 \mu \mathrm{m})$. Strip

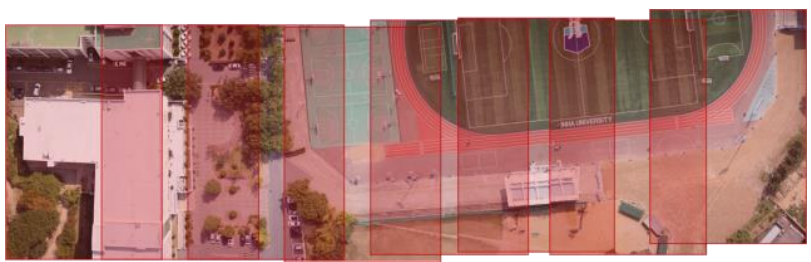

(a) Strip1: 8 images with narrow overlaps

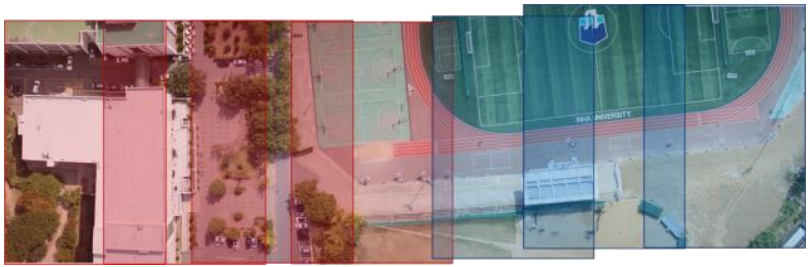

(b) Strip2: 7 images with very narrow overlaps

Figure 3. Two strips of UAV images with narrow overlaps. 
1 in Figure 3 was obtained with overlaps of about 30 40\%. Strip 2 was constituted with overlaps less than about $20 \%$.

For estimating relative transformation, tiepoints were extracted by FAST (Features from Accelerated Segment Test) and ORB (Oriented FAST and Rotated BRIEF) as detector and descriptor, respectively (Rosten and Drummond, 2006; Rublee et al., 2011). Outliers among the auto-extracted tiepoints were filtered by RANSAC (RANdom SAmple Consensus) algorithm (Fischler and Bolles, 1981). For radiometric correction, simple gain compensation and seamline extraction based on MAGDS (Minimum Absolute Gray Difference Sum) algorithm were carried out (Milgram, 1975; Shiren and Peng, 1989).

After global transformations of each image calculated from relative transformation relationships, bundle adjustment is generally performed for minimizing accumulated errors. However, in our condition, reliable bundle adjustment was hard to be performed because of weak geometry by narrow overlaps. For this reason, additional optimization with respect to initial estimations were not carried out.

For quantitative analysis, reprojection errors were measured. These were calculated as reversibility by forward and backward projection of observation points. Figure 4 shows reprojection errors of the two strip images caused by applying each relative transformation model. The experiment results showed that affine transformation and adjusted coplanar relative orientation were superior to homography and standard coplanar relative orientation and that this tendency was remarkably appeared with narrower overlaps between strip images. In the second strip with very narrow overlaps, homography based mosaicking did not work. These results indicate that rigorous models such as homography and standard coplanar relative orientation may not be suitable in case of image mosaicking for narrowly overlapping images.
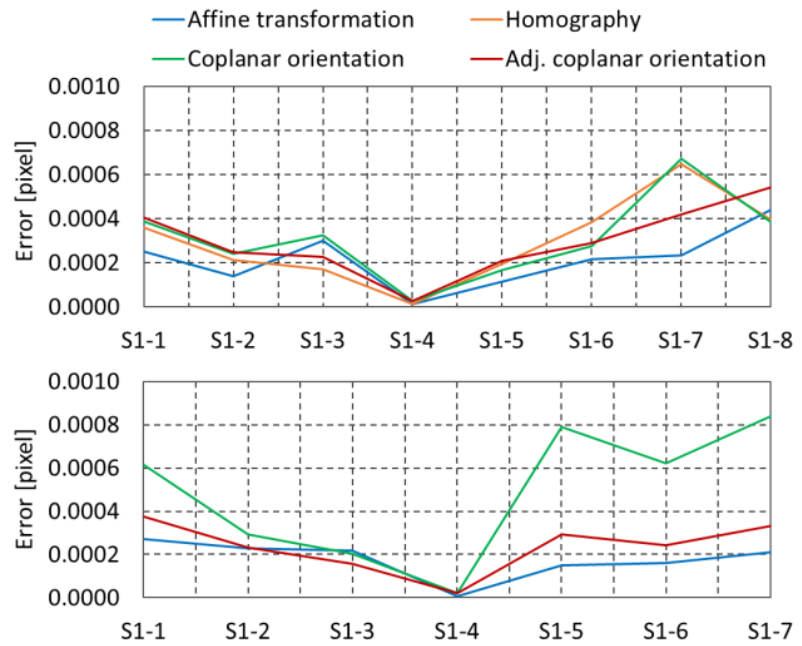

Figure 4. Reprojection errors for each transformation model (Top: Strip1 result, Bottom: Strip2 result).

These results were also confirmed on the mosaicked images in Figure 5 and 6 . In strip 1, homography and coplanar relative orientation model represented distortions. In strip 2, homography model was even worked and standard coplanar relative orientation also showed abnormal mosaicking result.

\section{CONCLUSION}

In this paper, we investigated a robust approach to generate a high quality mosaicked image from narrowly overlapping UAV images. Since quality of mosaicked image dependent to geometric correction, robust approach was discussed focused on transformation model. In order to investigate suitable transformation model for mosaicking of narrowly overlapping images, affine transformation, homography, standard coplanar relative orientation and adjusted coplanar relative orientation model were discussed and evaluated from two image strips with narrow overlaps.

The experiment result showed that existing homography and standard coplanar relative orientation model widely used for image mosaicking may not be suitable in case of images with narrow overlaps and that affine transformation model and adjusted coplanar relative orientation model can be applied instead of existing ones.

In future work, we will investigate constrained relative orientation for enhancing geometric accuracy of image mosaicking and bundle adjustments of each relative transformation model for optimal global transformation.

\section{REFERENCES}

Brown, M. and Lowe, D.G., 2007. Automatic panoramic image stitching using invariant features. International Journal of Computer Vision, 74(1), pp.59-73.

Fischler, M.A. and Bolles, R.C., 1981. Random sample consensus: a paradigm for model fitting with applications to image analysis and automated cartography. Communications of the ACM, 24, pp. 381-395.

Milgram, D.L., 1975. Computer Methods for Creating Photomosaics, IEEE Transactions on Computer, C-24(11), pp. 11131119.

Rosten, E. and Drummond, T., 2006. Machine learning for highspeed corner detection, In: 9th European Conference on Computer Vision, Graz, Austria, Part I, pp. 430-443.

Rublee, E., Rabaud, V., Konolige, K. and Gradski, K., 2011. ORB: an efficient alternative to SIFT or SURF, In: IEEE International Conference on Computer Vision, pp. 2564-2571.

Shiren, Y., Li, L., and Peng, G., 1989. Two-Dimensional SeamPoint Searching in Digital Image Mosaicing, Photogrammetric Engineering \& Remote Sensing, 55(1), pp. 49-53. 


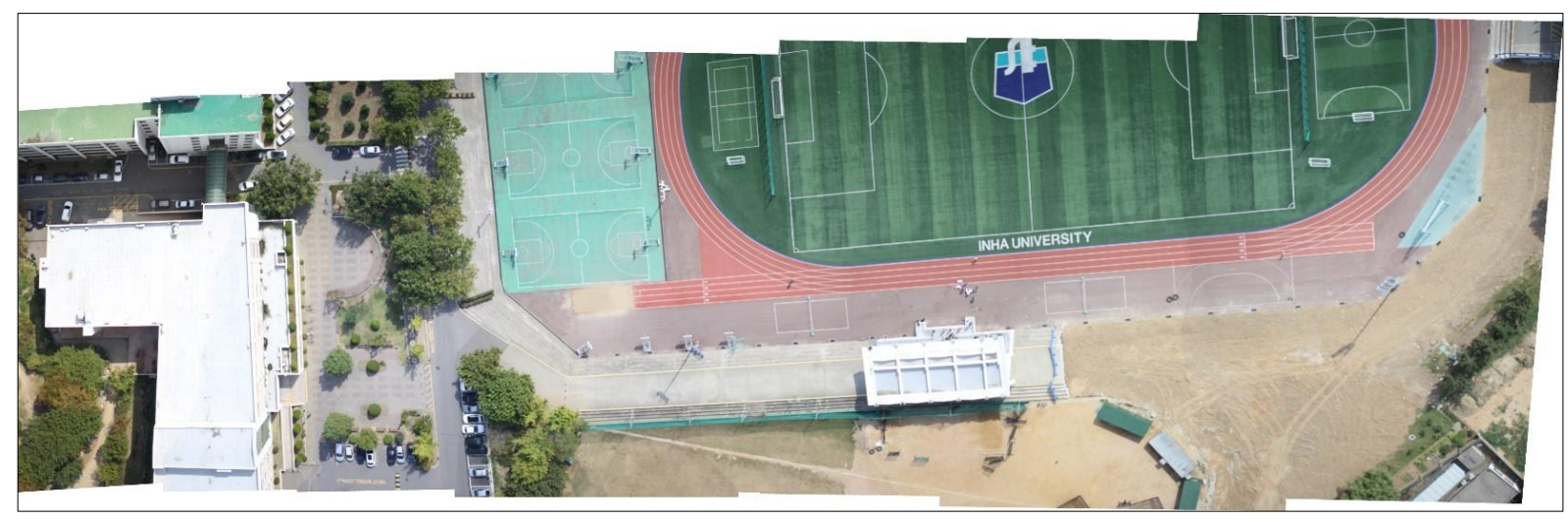

(a) Affine transformation model

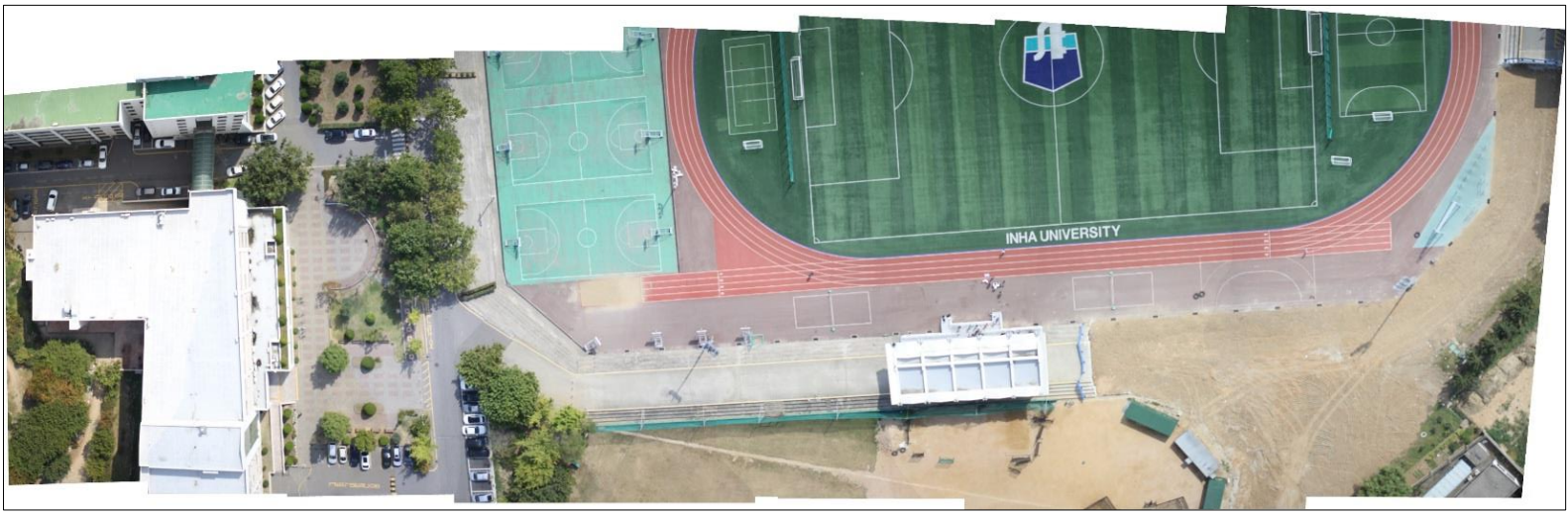

(b) Homography model

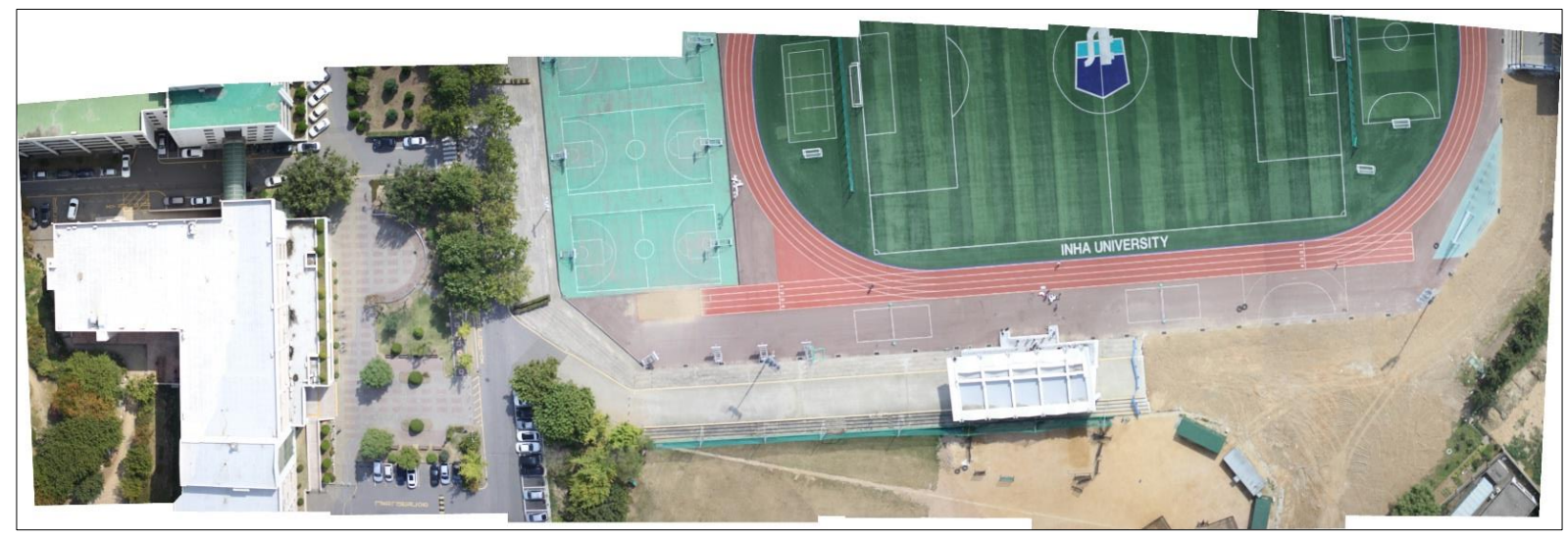

(c) Coplanar orientation model

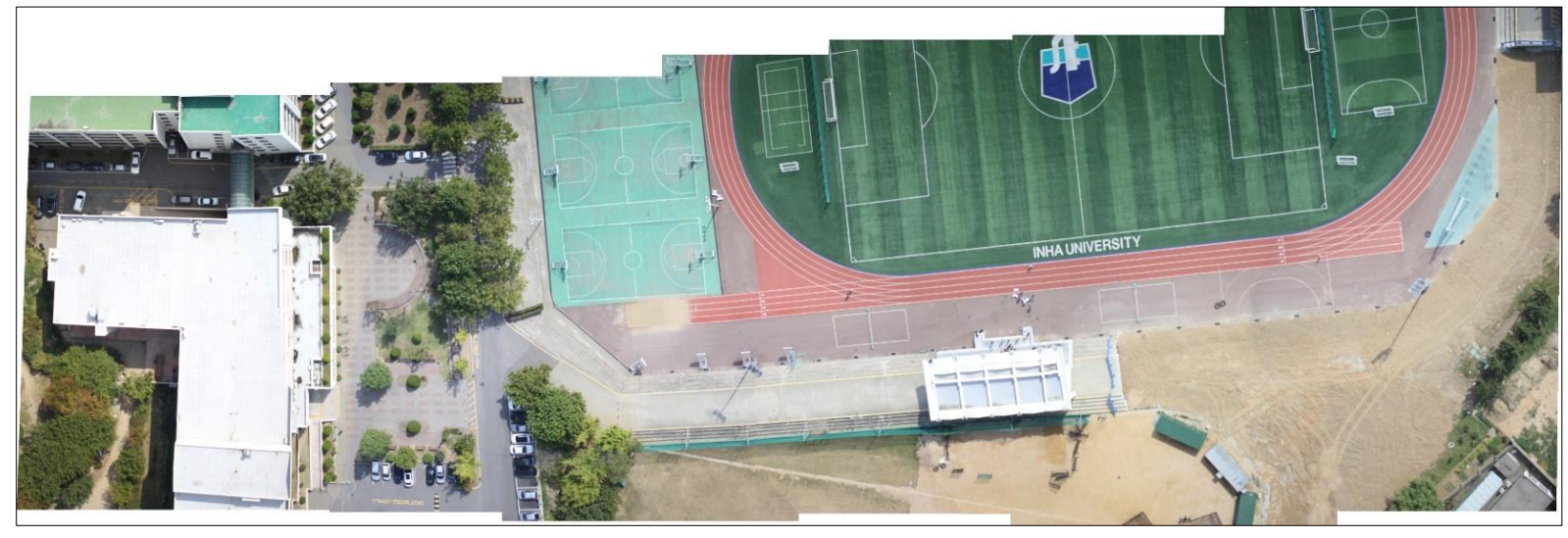

(d) Adjusted coplanar orientation model

Figure 5. Image mosaicking result of strip 1. 


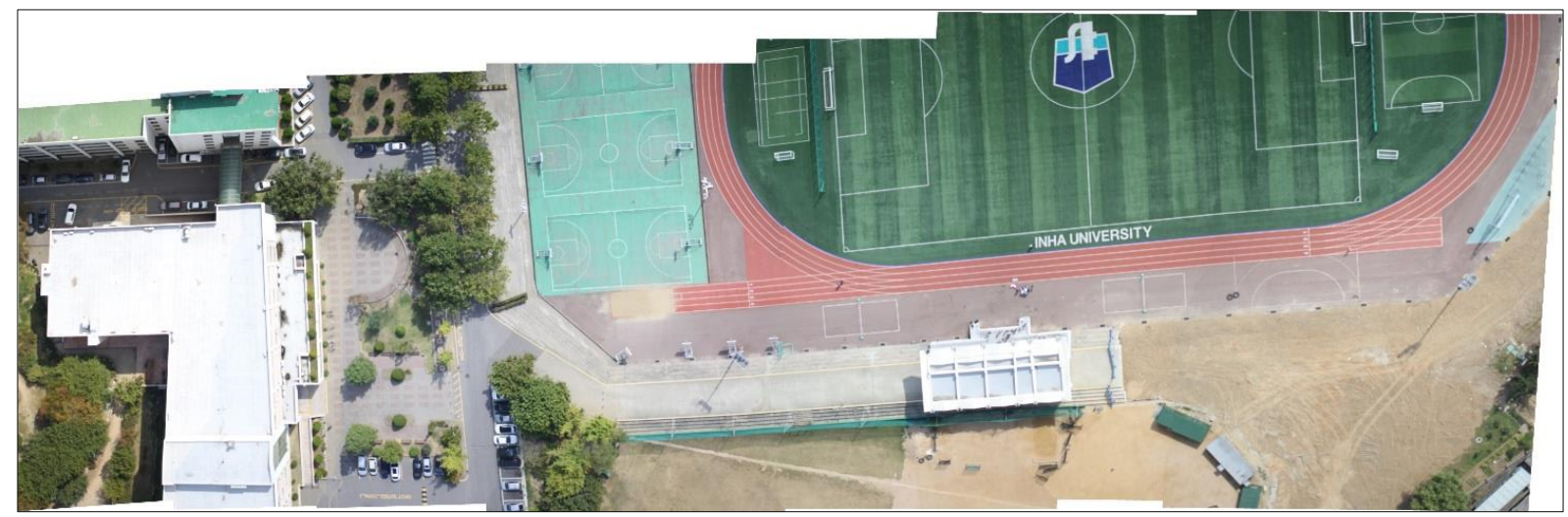

(a) Affine transformation model

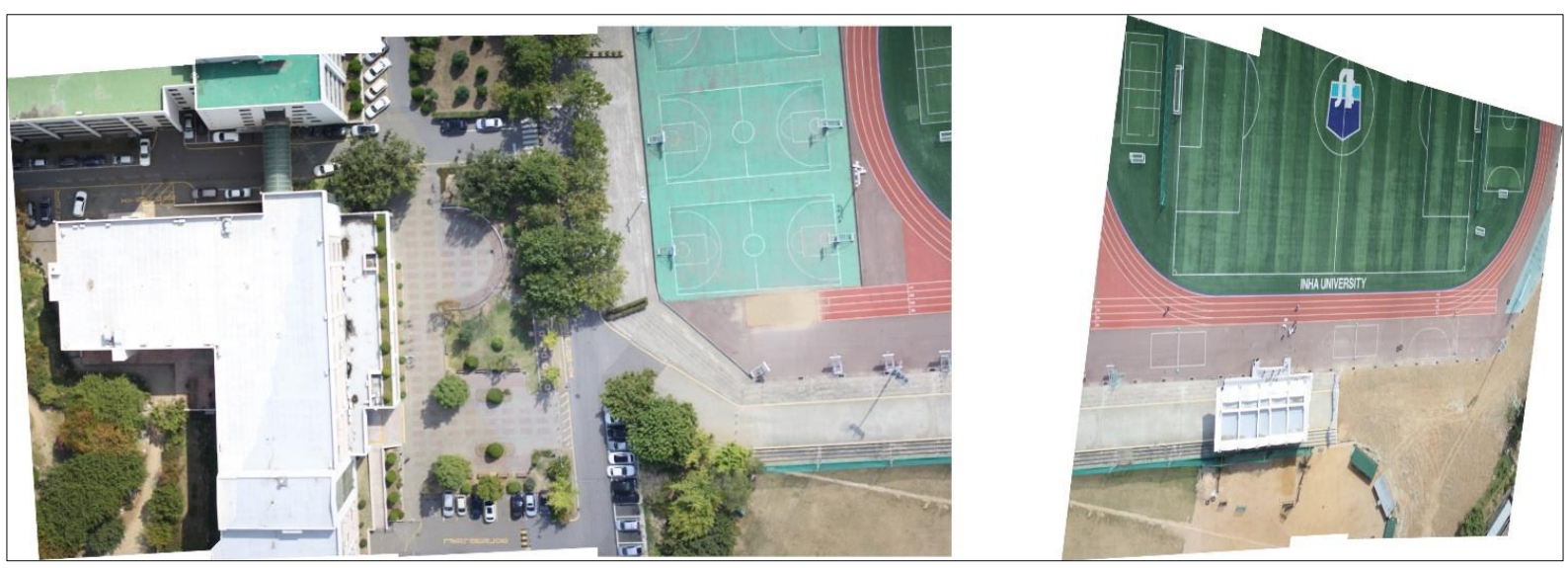

(b) Coplanar orientation model

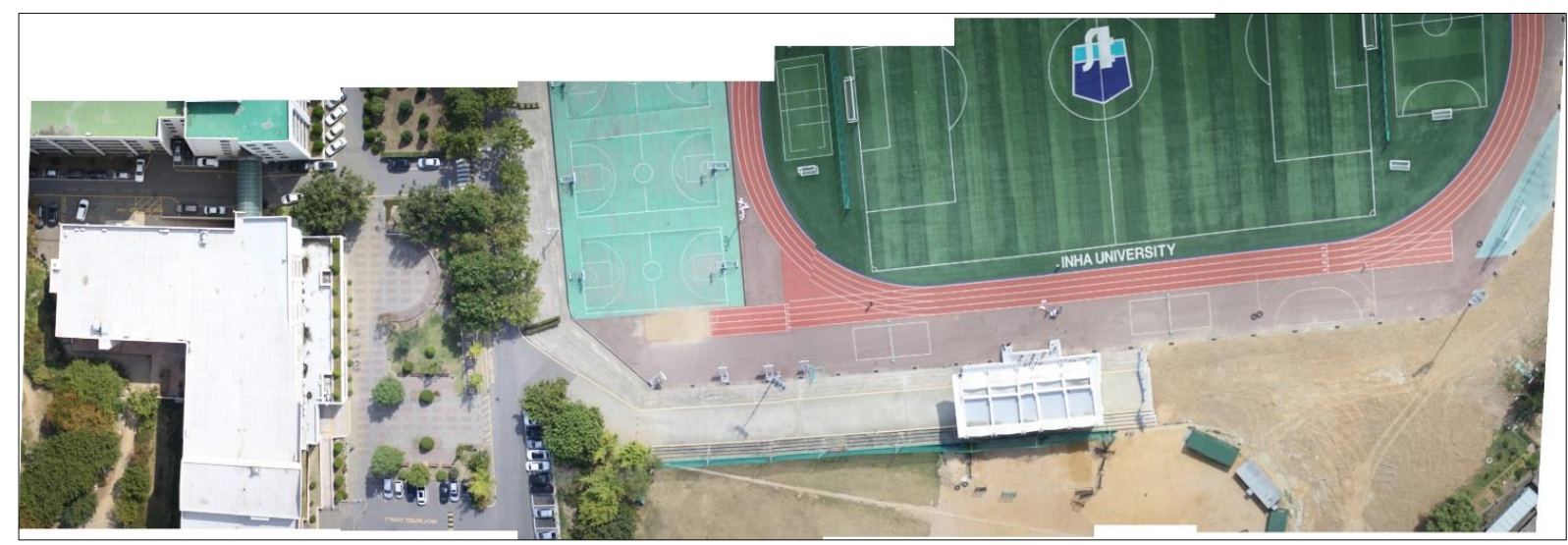

(c) Adjusted coplanar orientation model

Figure 6. Image mosaicking result of strip 2. 